\title{
Diagonal Lyapunov-Krasovskii functionals for discrete-time positive systems with delay
}

\author{
A.Yu. Aleksandrov ${ }^{\mathrm{b}}$, Oliver Mason ${ }^{\mathrm{a}, *}$ \\ a Hamilton Institute, National University of Ireland, Maynooth, Maynooth, Co. Kildare, Ireland \\ ${ }^{\mathrm{b}}$ Faculty of Applied Mathematics and Control Processes, St. Petersburg State University, 35 Universitetskij Pr., 198504 Petrodvorets, St. Petersburg, Russia
}

\section{H I G H L I G H T S}

- Conditions for diagonal Lyapunov-Krasovskii functional existence are given.

- The connection between diagonal L-K functionals and absolute stability is shown.

- The previous facts are extended to nonlinear and switched systems.

\section{A R T I C L E I N F O}

\section{Article history:}

Received 20 December 2012

Received in revised form 10 June 2013

Accepted 28 October 2013

Available online 6 December 2013

\section{Keywords:}

Positive systems

Delay

Stability

Diagonal Lyapunov-Krasovskii functional

\begin{abstract}
A B S T R A C T
We consider the existence of diagonal Lyapunov-Krasovskii (L-K) functionals for positive discrete-time systems subject to time-delay. In particular, we show that the existence of a diagonal functional is necessary and sufficient for delay-independent stability of a positive linear time-delay system. We extend this result and provide conditions for the existence of diagonal $\mathrm{L}-\mathrm{K}$ functionals for classes of nonlinear positive time-delay systems, which are not necessarily order preserving. We also describe sufficient conditions for the existence of common diagonal L-K functionals for switched positive systems subject to time-delay.
\end{abstract}

(c) 2013 Elsevier B.V. All rights reserved.

\section{Introduction}

Positive systems, in which the state variables remain nonnegative for all time given nonnegative initial conditions, are of importance in modeling a wide variety of applications; particularly in domains such as Communications, Biology, Ecology and Economics. There is now a well-developed and understood theory of positive linear time-invariant (LTI) systems [1,2]. Motivated by the simple fact that realistic models must incorporate factors such as nonlinearity, time-delay and time-varying parameters, several authors have recently worked on extending aspects of the theory of positive LTI systems to more realistic and general system classes [3-8]. The work of the current paper continues in this vein.

Specifically, we consider classes of discrete time positive systems and the impact of time-delay on their stability properties. We first note that a recently published result on the existence of diagonal L-K functionals for positive linear systems in continuous time admits a natural analogue in discrete time. In fact, we explicitly describe how to construct such functionals in the discrete time case.

\footnotetext{
* Corresponding author. Tel.: +3530 017086274 ; fax: +353 017086269 .

E-mail address: oliver.mason@nuim.ie (O. Mason).
}

This result, as in [9], shows that a fundamental property of positive LTI systems, namely diagonal stability, extends to positive linear time-delay systems in discrete time.

In Section 4, we use the results in Section 3 to derive a closely related condition for positive systems with sector-bounded nonlinearities to be stable independent of delay. It is worth noting that this result applies to nonlinear positive systems that are not necessarily order preserving, in contrast to others in the literature. It is closely related to various results for systems of Persidskii type described in [10] and elsewhere. As noted in this reference, these systems arise in applications such as digital filtering. We then turn our attention to nonlinear switched positive systems with timedelay in Section 5. Finally, in Section 6, we present some brief concluding remarks.

\section{Notation and background}

Throughout the paper $\mathbb{R}^{n}$ and $\mathbb{R}^{n \times n}$ denote the vector spaces of $n$-tuples of real numbers and of $n \times n$ matrices respectively. For vectors $v \in \mathbb{R}^{n}, v \geq 0$ means $v_{i} \geq 0$ for $1 \leq i \leq n, v \gg 0$ means $v_{i}>$ 0 for $1 \leq i \leq n$. We use the notation $A^{T}$ for the transpose of a matrix $A$ and $P \succ 0$ to denote that the matrix $P$ is positive definite. The 
identity matrix is denoted by $I$; the dimension will be clear in context. We say that a matrix $A \in \mathbb{R}^{n \times n}$ is nonnegative if all of its elements are nonnegative. The matrix $A$ is Schur if all of its eigenvalues have modulus strictly less than 1 .

The following known facts about nonnegative matrices are useful for our later results.

Proposition 2.1. Let $A \in \mathbb{R}^{n \times n}$ be nonnegative. The following are true.

(i) $A$ is Schur if and only if there exists some $v \gg 0$ with $A v \ll v$.

(ii) If $A v \ll v$ and $A^{T} w \ll w$ then defining

$$
D=\operatorname{diag}\left(w_{i} / v_{i}\right)
$$

we have

$A^{T} D A-D \prec 0$.

\section{Diagonal L-K functionals for linear discrete time delayed systems}

Consider the positive linear time-delay system

$\dot{x}(t)=A x(t)+B x(t-\tau)$

where $A$ is Metzler and $B$ is nonnegative. It is now well known that (1) is asymptotically stable for all values of $\tau \geq 0$ if and only if $A+B$ is Hurwitz [11], meaning that all of its eigenvalues lie in the open left half of the complex plane.

In the recent paper [9], the following fact was established.

Theorem 3.1. The positive linear time-delay system (1) has a $L-K$ functional of the form

$x(t)^{T} P x(t)+\int_{-\tau}^{0} x(t+s) Q x(t+s) d s$

where $P$ and $Q$ are positive definite matrices and $P$ is diagonal, if and only if the matrix $A+B$ is Hurwitz stable.

In the current section, we derive a discrete time version of Theorem 3.1.

Formally, consider the system

$x(k+1)=A x(k)+B_{1} x(k-1)+\cdots+B_{l} x(k-l)$,

where $A, B_{1}, \ldots, B_{l}$ are nonnegative matrices in $\mathbb{R}^{n \times n}$. Under these assumptions (2) defines a positive time-delay system in discrete time, meaning that if initial conditions $x(-l), \ldots, x(0)$ are nonnegative then $x(k) \geq 0$ for all $k \geq 0$. For notational convenience, we write $x^{(k)}$ for the augmented state vector $x^{(k)}=(x(k), x(k-1)$, $\ldots, x(k-l))^{T}$ in $\mathbb{R}^{(l+1) n}$.

In the following result, we present a direct argument to establish a discrete-time version of Theorem 3.1.

Theorem 3.2. Consider the system (2) and assume that the matrix $S$ given by

$S=A+B_{1}+\cdots+B_{l}$

is Schur. Then there exists a $L-K$ functional for (2) of the form

$$
\begin{aligned}
V\left(x^{(k)}\right)= & x^{T}(k) P x(k)+x^{T}(k-1) Q_{1} x(k-1) \\
& +\left\{x^{T}(k-1) Q_{2} x(k-1)+x^{T}(k-2) Q_{2} x(k-2)\right\} \\
& +\cdots+\left\{x^{T}(k-1) Q_{1} x(k-1)+x^{T}(k-2)\right. \\
& \left.\times Q_{l} x(k-2)+\cdots+x^{T}(k-l) Q_{1} x(k-l)\right\}
\end{aligned}
$$

Proof. We write $P=\operatorname{diag}\left\{p_{1}, \ldots, p_{n}\right\}, Q_{s}=\operatorname{diag}\left\{\mu_{s 1}, \ldots, \mu_{s n}\right\}$ for the matrices appearing in (4). We shall show that it is possible to find positive real numbers $p_{i}$ and $\mu_{s i}, s=1, \ldots, l, i=1, \ldots, n$ such that (4) is a L-K functional for the system (2).

Consider the difference, $\Delta V=V\left(x^{(k+1)}\right)-V\left(x^{(k)}\right)$ of the functional (4) along trajectories of the system (2). We obtain

$$
\begin{aligned}
\Delta V= & x^{T}(k)\left(A^{T} P A-P+\sum_{r=1}^{l} Q_{r}\right) x(k) \\
& -\sum_{r=1}^{l} x^{T}(k-r) Q_{r} x(k-r)+2 x^{T}(k) A^{T} P \sum_{r=1}^{l} B_{r} x(k-r) \\
& +\left(\sum_{r=1}^{l} B_{r} x(k-r)\right)^{T} P \sum_{r=1}^{l} B_{r} x(k-r) .
\end{aligned}
$$

Expanding this yields

$$
\begin{aligned}
\Delta V= & \sum_{r=1}^{l} \sum_{j=1}^{n} \mu_{r j} x_{j}^{2}(k)-\sum_{j=1}^{n} p_{j} x_{j}^{2}(k) \\
& -\sum_{r=1}^{l} \sum_{j=1}^{n} \mu_{r j} x_{j}^{2}(k-r)+\sum_{i, j=1}^{n} x_{i}(k) x_{j}(k) \sum_{m=1}^{n} p_{m} a_{m i} a_{m j} \\
& +2 \sum_{r=1}^{l} \sum_{i, j=1}^{n} x_{i}(k) x_{j}(k-r) \sum_{m=1}^{n} p_{m} a_{m i} b_{m j}^{(r)} \\
& +\sum_{r, s=1}^{l} \sum_{i, j=1}^{n} x_{i}(k-r) x_{j}(k-s) \sum_{m=1}^{n} p_{m} b_{m i}^{(r)} b_{m j}^{(s)} .
\end{aligned}
$$

The expression on the right hand side above defines a quadratic form

$$
\begin{aligned}
W= & \sum_{r=1}^{l} \sum_{j=1}^{n} \mu_{r j} x_{j}^{2}-\sum_{j=1}^{n} p_{j} x_{j}^{2}-\sum_{r=1}^{l} \sum_{j=1}^{n} \mu_{r j} x_{r j}^{2} \\
& +\sum_{i, j=1}^{n} x_{i} x_{j} \sum_{m=1}^{n} p_{m} a_{m i} a_{m j}+2 \sum_{r=1}^{l} \sum_{i, j=1}^{n} x_{i} x_{r j} \\
& \times \sum_{m=1}^{n} p_{m} a_{m i} b_{m j}^{(r)}+\sum_{r, s=1}^{l} \sum_{i, j=1}^{n} x_{r i} x_{s j} \sum_{m=1}^{n} p_{m} b_{m i}^{(r)} b_{m j}^{(s)}
\end{aligned}
$$

in the variables $x_{i}, 1 \leq i \leq n, x_{r i}, 1 \leq i \leq n, 1 \leq r \leq l$. Our goal is to show that there exist positive numbers $p_{i}$ and $\mu_{s i}, s=1, \ldots, l$, $i=1, \ldots, n$, for which the quadratic form $W$ is negative definite.

As the matrix $S$ is Schur, it follows from Proposition 2.1 that there exist positive vectors $\theta=\left(\theta_{1}, \ldots, \theta_{n}\right)^{T}$ and $d=\left(d_{1}, \ldots\right.$, $\left.d_{n}\right)^{T}$ satisfying the inequalities

$$
(S-I) \theta \ll 0, \quad(S-I)^{T} d \ll 0 .
$$

Write $\omega=(S-I) \theta, \psi=(S-I)^{T} d$, and note that the vectors $\omega$ and $\psi$ are entrywise negative.

If we write, $p_{i}=d_{i} / \theta_{i}, x_{i}=\theta_{i} z_{i}, x_{r i}=\theta_{i} v_{r i}$, then we can rewrite $W$ as

$$
\begin{aligned}
W= & \sum_{r=1}^{l} \sum_{j=1}^{n} \mu_{r j} \theta_{j}^{2} z_{j}^{2}-\sum_{j=1}^{n} d_{j} \theta_{j} z_{j}^{2}-\sum_{r=1}^{l} \sum_{j=1}^{n} \mu_{r j} \theta_{j}^{2} v_{r j}^{2} \\
& +\sum_{i, j=1}^{n} \theta_{i} z_{i} \theta_{j} z_{j} \sum_{m=1}^{n} \frac{d_{m}}{\theta_{m}} a_{m i} a_{m j}+2 \sum_{r=1}^{l} \sum_{i, j=1}^{n} \theta_{i} z_{i} \theta_{j} v_{r j} \\
& \times \sum_{m=1}^{n} \frac{d_{m}}{\theta_{m}} a_{m i} b_{m j}^{(r)}+\sum_{r, s=1}^{l} \sum_{i, j=1}^{n} \theta_{i} v_{r i} \theta_{j} v_{s j} \sum_{m=1}^{n} \frac{d_{m}}{\theta_{m}} b_{m i}^{(r)} b_{m j}^{(s)} .
\end{aligned}
$$

where $P, Q_{1}, \ldots, Q_{l}$ are positive definite diagonal matrices. 
Now using the inequalities $2 z_{i} z_{j} \leq z_{i}^{2}+z_{j}^{2}, 2 z_{i} v_{r j} \leq z_{i}^{2}+v_{r j}^{2}$, $2 v_{r i} v_{s j} \leq v_{r i}^{2}+v_{s j}^{2}$ and grouping terms appropriately, we can see that

$$
\begin{aligned}
W \leq & \sum_{r=1}^{l} \sum_{j=1}^{n} \mu_{r j} \theta_{j}^{2} z_{j}^{2}-\sum_{j=1}^{n} d_{j} \theta_{j} z_{j}^{2}-\sum_{r=1}^{l} \sum_{j=1}^{n} \mu_{r j} \theta_{j}^{2} v_{r j}^{2} \\
& +\sum_{j=1}^{n} \theta_{j} z_{j}^{2} \sum_{i, m=1}^{n} \frac{d_{m}}{\theta_{m}} \theta_{i} s_{m i} a_{m j}+\sum_{r=1}^{l} \sum_{j=1}^{n} \theta_{j} v_{r j}^{2} \sum_{i, m=1}^{n} \theta_{i} \frac{d_{m}}{\theta_{m}} b_{m j}^{(r)} s_{m i} .
\end{aligned}
$$

Here $s_{m i}$ are the entries of the matrix $S$.

It follows readily from (5) that

$$
\begin{aligned}
W \leq & \sum_{j=1}^{n} \theta_{j} z_{j}^{2}\left(\sum_{r=1}^{l} \mu_{r j} \theta_{j}-d_{j}+\sum_{m=1}^{n} d_{m} a_{m j}\right) \\
& +\sum_{r=1}^{l} \sum_{j=1}^{n} \theta_{j} v_{r j}^{2}\left(\sum_{m=1}^{n} d_{m} b_{m j}^{(r)}-\mu_{r j} \theta_{j}\right) .
\end{aligned}
$$

Now for $j=1, \ldots, n, r=1, \ldots, l$, and any $\varepsilon>0$, we can choose $\mu_{r j}>0$ such that

$\theta_{j}^{2} \mu_{r j}=\varepsilon+\theta_{j} \sum_{m=1}^{n} d_{m} b_{m j}^{(r)}$.

Substituting this into (6), we see that

$$
\begin{aligned}
W & \leq-\varepsilon \sum_{r=1}^{l} \sum_{j=1}^{n} v_{r j}^{2}+\varepsilon l \sum_{j=1}^{n} z_{j}^{2}+\sum_{j=1}^{n} \theta_{j} z_{j}^{2}\left(\sum_{m=1}^{n} d_{m} s_{m j}-d_{j}\right) \\
& =-\varepsilon \sum_{r=1}^{l} \sum_{j=1}^{n} v_{r j}^{2}+\varepsilon l \sum_{j=1}^{n} z_{j}^{2}+\sum_{j=1}^{n} \theta_{j} z_{j}^{2} \psi_{j} .
\end{aligned}
$$

It now follows readily that for $\varepsilon$ sufficiently small,

$W \leq-\beta \sum_{j=1}^{n} z_{j}^{2}-\varepsilon \sum_{r=1}^{l} \sum_{j=1}^{n} v_{r j}^{2}$

where $\beta$ is a positive constant. This completes the proof.

Remarks. The approach we have taken in proving Theorem 3.2 is an extension of the approaches suggested in $[12,13]$. It is also possible to prove this result by suitably adapting the methods of [14] and writing (2) in the higher-dimensional, companion-matrix form. This approach has been taken in [15] in which applications to stabilization are also discussed. It is worth noting the arguments presented in [16] in favor of working in the state space $\mathbb{R}^{n}$, as opposed to the higher-dimensional space.

Theorem 3.1 establishes the existence of a $\mathrm{L}-\mathrm{K}$ functional in which the matrix $Q$ is positive definite. In Theorem 3.2, we arrive at the stronger conclusion that each $Q_{i}$ can be taken to be diagonal. In addition to providing a simpler form for the functional, Theorem 3.2 also describes a direct, constructive approach for obtaining the functional.

The following corollary is now practically immediate.

Corollary 3.1. Consider the system

$x(k+1)=A x(k)+B_{1} x\left(k-m_{1}\right)+\cdots+B_{l} x\left(k-m_{l}\right)$,

where $A, B_{1}, \ldots, B_{l}$ are nonnegative matrices, and $m_{1}, \ldots, m_{l}$ are nonnegative integer delays. The system (7) is asymptotically stable for any values of $m_{1}, \ldots, m_{l}$ if and only if there exists a diagonal $L-K$ functional of the form (4) for (7).

Proof. The sufficiency follows directly from Theorem 3.2. For necessity, note that system (7) is by assumption also stable for the case where $m_{1}=\cdots=m_{l}=0$.
Remark. As stated, the above result appears to require that a particular one of the system matrices, namely $A$, multiplies the undelayed current state. Of course, the condition on the matrix $S$ is invariant under permutations of the matrices $A, B_{1}, \ldots, B_{l}$ and the result also holds for systems of the form

$x(k+1)=A x\left(k-m_{0}\right)+B_{1} x\left(k-m_{1}\right)+\cdots+B_{l} x\left(k-m_{l}\right)$,

where $m_{0}, \ldots, m_{l}$ are nonnegative integer delays. This follows from the statement of the corollary as can be seen by taking $A$ as the zero matrix. Finally, note that the equivalence of the stability of (7) with the matrix $S$ (3) being Schur stable is known [17].

\section{Delay independent stability for nonlinear positive systems}

We next present a straightforward extension of Theorem 3.2 to a class of nonlinear positive systems. The class we consider is closely related to continuous time systems of Persidskii type [10] and are motivated by applications such as digital filtering.

Formally, consider a diagonal nonlinearity $f: \mathbb{R}^{n} \rightarrow \mathbb{R}^{n}, f\left(x_{1}\right.$, $\left.\ldots, x_{n}\right)=\left(f_{1}\left(x_{1}\right), f_{2}\left(x_{2}\right), \ldots, f_{n}\left(x_{n}\right)\right)^{T}$ where each $f_{i}$ is continuous and satisfies the following conditions:

$x_{i} f_{i}\left(x_{i}\right)>0$ for $x_{i} \neq 0$;

$\left|f_{i}\left(x_{i}\right)\right| \leq\left|x_{i}\right|$.

Consider the system

$x(k+1)=A f(x(k))+B_{1} f(x(k-1))+\cdots+B_{l} f(x(k-l))$

where $A, B_{1}, \ldots, B_{l}$ are nonnegative matrices. Conditions (8), (9) ensure that (10) is a positive system with an equilibrium at the origin. However, in our next result, it is not necessary to restrict initial conditions to the positive orthant.

Theorem 4.1. Let $S$ given by (3) be a Schur matrix. Then there exists a $L-K$ functional of the form

$$
\begin{aligned}
V\left(x^{(k)}\right)= & x^{T}(k) P x(k)+f^{T}(x(k-1)) Q_{1} f(x(k-1)) \\
& +\left\{f^{T}(x(k-1)) Q_{2} f(x(k-1))+f^{T}(x(k-2))\right. \\
& \left.\times Q_{2} f(x(k-2))\right\}+\cdots+\left\{f^{T}(x(k-1)) Q_{l} f\right. \\
& \left.\times(x(k-1))+\cdots+f^{T}(x(k-l)) Q_{l} f(x(k-l))\right\}
\end{aligned}
$$

where $P, Q_{1}, \ldots, Q_{l}$ are positive definite diagonal matrices.

Proof. As in the proof of Theorem 3.2, we write $P=\operatorname{diag}\left\{p_{1}, \ldots\right.$, $\left.p_{n}\right\}, Q_{s}=\operatorname{diag}\left\{\mu_{s 1}, \ldots, \mu_{s n}\right\}$ for $s=1, \ldots, l, i=1, \ldots, n$.

Now consider the difference $\Delta V=V\left(x^{(k+1)}\right)-V\left(x^{(k)}\right)$ of the functional (11) along trajectories of the system (10). By direct calculation, we see that

$$
\begin{aligned}
\Delta V= & \left(A f(x(k))+\sum_{r=1}^{l} B_{r} f(x(k-r))\right)^{T} \\
& \times P\left(A f(x(k))+\sum_{r=1}^{l} B_{r} f(x(k-r))\right) \\
& -x^{T}(k) P x(k)+f^{T}(x(k)) \sum_{r=1}^{l} Q_{r} f(x(k)) \\
& -\sum_{r=1}^{l} f^{T}(x(k-r)) Q_{r} f(x(k-r)) .
\end{aligned}
$$

From (8) and (9), it follows that

$\Delta V \leq f^{T}(x(k))\left(A^{T} P A-P+\sum_{r=1}^{l} Q_{r}\right) f(x(k))$ 


$$
\begin{aligned}
& -\sum_{r=1}^{l} f^{T}(x(k-r)) Q_{r} f(x(k-r)) \\
& +2 f^{T}(x(k)) A^{T} P \sum_{r=1}^{l} B_{r} f(x(k-r)) \\
& +\left(\sum_{r=1}^{l} B_{r} f(x(k-r))\right)^{T} P\left(\sum_{r=1}^{l} B_{r} f(x(k-r))\right) .
\end{aligned}
$$

As in the proof of Theorem 3.2, the right hand side above is a quadratic form $W$ in $f_{i}\left(x_{i}(k)\right), f_{i}\left(x_{i}(k-r)\right)$ for $1 \leq i \leq n, 1 \leq r \leq l$. Writing $p_{i}=\frac{d_{i}}{\theta_{i}}, f_{i}\left(x_{i}(k)\right)=\theta_{i} z_{i}, f_{i}\left(x_{i}(k-r)\right)=\theta_{i} v_{r i}$, we can apply arguments identical to those used in Theorem 3.2 to show that (6) holds. In our current case the same arguments used in the final part of this previous theorem, imply that there exist positive numbers $p_{i}$ and $\mu_{s i}, s=1, \ldots, l, i=1, \ldots, n$ and some positive $\beta$ such that

$W \leq-\beta\left(f^{T}(x(k)) f(x(k))+\sum_{r=1}^{l} f^{T}(x(k-r)) f(x(k-r))\right)$

holds. This completes the proof.

Remark. Theorem 3.4.4 of [10] provides a diagonal Lyapunov stability condition for nonlinear systems without delay. In the discussion beneath Theorem 3.4.4 of [10], a result for linear systems with delay is described. Theorem 4.1 takes both nonlinearity and delay into account. While the system class covered in Theorem 4.1 is not necessarily order-preserving, it should be noted that the restriction $\left|f_{i}\left(x_{i}\right)\right| \leq\left|x_{i}\right|$ means that the stability of (10) follows from the stability of the linear comparison system obtained by setting $f_{i}\left(x_{i}\right)=x_{i}$, which is order-preserving. The novelty in Theorem 4.1 is the fact that a diagonal-type L-K functional exists for the system in this case.

Finally, note that it is straightforward to derive a version of Corollary 3.1 for the system class (10).

\section{Switched positive systems with time-delay}

Our goal in this section is to further extend the analysis of the previous two sections to switched nonlinear positive systems constructed from constituent systems of the form (10). We first consider the single delay case in the interest of notational simplicity and clarity.

\subsection{A single delay} form

In this subsection, we shall consider switched systems of the

$x(k+1)=A_{\sigma(k)} f(x(k))+B_{\sigma(k)} f(x(k-l))$

corresponding to sets $\left\{A^{(1)}, \ldots, A^{(N)}\right\},\left\{B^{(1)}, \ldots, B^{(N)}\right\}$ of nonnegative matrices. The nonlinearity $f(x)=\left(f_{1}\left(x_{1}\right), \ldots, f_{n}\left(x_{n}\right)\right)^{T}$ satisfies the conditions (8), (9). The switching signal $\sigma$ maps the nonnegative integers into $\{1, \ldots, N\}$ and selects which constituent system is active at each time $k$.

The results of Section 3 imply that if a diagonal Lyapunov function exists for the undelayed system obtained from (2) by setting all delays equal to 0 , then this implies that a diagonal L-K functional also exists for (2) for all nonnegative integer-valued delays. It is tempting to conjecture that in the switched case, if a common diagonal Lyapunov function exists for the switched system with zero delay, then a common diagonal L-K functional exists for the delayed system also. Unfortunately this is not the case as is shown by the following example.
Example 5.1. Consider the system

$x(k+1)=A_{\sigma(k)} x(k)+B_{\sigma(k)} x(k-1), \quad \sigma(\cdot) \in\{1,2\}$,

where:

$\begin{array}{ll}A_{1}=\left(\begin{array}{cc}0.06 & 0.2 \\ 0.27 & 0.21\end{array}\right), & B_{1}=\left(\begin{array}{cc}0.66 & 0.08 \\ 0.13 & 0.2\end{array}\right) ; \\ A_{2}=\left(\begin{array}{cc}0.07 & 0.19 \\ 0.4 & 0.11\end{array}\right), & B_{2}=\left(\begin{array}{ll}0.27 & 0.21 \\ 0.29 & 0.47\end{array}\right) .\end{array}$

It can be verified using the MATLAB LMI toolbox that the undelayed system (with system matrices $A_{1}+B_{1}, A_{2}+B_{2}$ ) has a common diagonal Lyapunov function.

On the other hand, the switched delayed system is not stable and hence certainly does not have a common diagonal Lyapunov functional; in fact it cannot possess a common Lyapunov functional of any form. To see this, consider the higher-dimensional companion form of the system in which the state vectors are $(x(k), x(k+$ 1)) $)^{T}$ and the system matrices are

$C_{i}=\left(\begin{array}{cc}0 & I \\ B_{i} & A_{i}\end{array}\right)$

for $i=1,2$. The spectral radius of the matrix $C_{1} C_{2}$ is 1.0027 . As this is strictly greater than 1 , the switched delayed system cannot be asymptotically stable and hence it cannot have a common Lyapunov functional.

In the next result, we provide a sufficient condition for the existence of a common L-K functional of the form (11) for the family of subsystems:

$x(k+1)=A^{(s)} f(x(k))+B^{(s)} f(x(k-l)), \quad s=1, \ldots, N$,

where $A^{(s)}, B^{(s)}$ are nonnegative matrices, $l$ is a nonnegative integer delay, and the nonlinearities $f(x)=\left(f_{1}\left(x_{1}\right), \ldots, f_{n}\left(x_{n}\right)\right)^{T}$ satisfy the conditions (8), (9).

Theorem 5.1. Let $A^{(s)}, B^{(s)}$ be nonnegative matrices for $s=1, \ldots$, $N$. Assume that there exist vectors $d \gg 0, \theta \gg 0$ satisfying the inequalities

$\left(A^{(s)}+B^{(r)}-I\right)^{T} d \ll 0, \quad s, r=1, \ldots, N$,

$\left(A^{(s)}+B^{(s)}-I\right) \theta \ll 0, \quad s=1, \ldots, N$.

Then there exists a common $L-K$ functional of the form (11) for (13).

Proof. Proceeding as in the proof of Theorems 3.2 and 4.1, we consider the difference $\Delta V=V\left(x^{(k+1)}\right)-V\left(x^{(k)}\right)$ of the functional (11) along trajectories of the system (13) for some $s$ in $\{1, \ldots, N\}$. Note that (13) can be viewed as a special case of (10) in which $B_{i}=0$ for all $i \neq l$. As before, writing $p_{i}=d_{i} / \theta_{i}, f_{i}\left(x_{i}(k)\right)=\theta_{i} z_{i}, f_{i}\left(x_{i}(k-r)\right)=$ $\theta_{i} v_{r i}, r=1, \ldots, l, i=1, \ldots, n$ and using (8), (9), we can show that

$$
\begin{aligned}
\Delta V \leq & \sum_{j=1}^{n} \theta_{j} z_{j}^{2}\left(\sum_{r=1}^{l} \mu_{r j} \theta_{j}-d_{j}+\sum_{m=1}^{n} d_{m} a_{m j}^{(s)}\right) \\
& +\sum_{j=1}^{n} \theta_{j} v_{l j}^{2}\left(\sum_{m=1}^{n} d_{m} b_{m j}^{(s)}-\mu_{l j} \theta_{j}\right)-\sum_{r=1}^{l-1} \sum_{j=1}^{n} \mu_{r j} \theta_{j}^{2} v_{r j}^{2} .
\end{aligned}
$$

Define $\Theta=\operatorname{diag}\left(\theta_{1}, \ldots, \theta_{n}\right)$. The last term in (16) will be negative for any choice of positive $\mu_{r j}$ for $1 \leq r \leq l-1,1 \leq j \leq n$.

We claim that there exist positive vectors $\mu \gg 0, \overline{\mu^{\prime}} \gg \mu$ such that

$$
\begin{aligned}
& \left(A^{(s)}-I\right)^{T} d+\Theta \mu^{\prime} \ll 0, \quad B^{(s)^{T}} d-\Theta \mu \ll 0, \\
& \quad s=1, \ldots, N .
\end{aligned}
$$


To see this, note that as there are only finitely many inequalities in (14), there exists some positive vector $w \gg 0$ with

$\left(A^{(s)}-I\right)^{T} d+\left(B^{(r)}\right)^{T} d \ll-w$

for $s, r$ in $\{1, \ldots, N\}$. Define $v=\max _{r}\left(B^{(r)}\right)^{T} d$ as the componentwise maximum of the vectors $\left(B^{(r)}\right)^{T} d$. It is now easy to verify that $\mu=\Theta^{-1}\left(v+\frac{w}{2}\right), \mu^{\prime}=\Theta^{-1}\left(v+\frac{3 w}{4}\right)$ (for example) satisfy (17).

If we now set $\mu_{l j}=\mu_{j}$ for $1 \leq j \leq n$ and $\mu_{r j}=\left(\mu_{j}^{\prime}-\mu_{j}\right) /(l-1)$ for $1 \leq r \leq l-1$, then (11) defines a L-K functional for (13) as claimed.

Remark. The previous result gives sufficient conditions for the existence of a common diagonal $\mathrm{L}-\mathrm{K}$ functional and there is a gap to necessity. It should be noted that the conditions are closely related to those that appear in Theorem 3 of [18]. This reference provides stability conditions for switched positive time-delay systems in continuous time of the form

$\dot{x}(t)=A_{\sigma(t)} x(t)+B_{\sigma(t)} x(t-\tau), \quad \sigma(\cdot) \in\{1, \ldots, l\}$.

The two sufficient conditions that appear in [18] imply the existence of a positive vector $\alpha$ such that $\left(A_{i}^{T}+B_{j}^{T}\right) \alpha \ll 0$ for all $i, j$, which is simply the continuous time analogue of our condition (14) above. In [18] however, it is the class of linear copositive Lyapunov functionals that is studied, rather than diagonal Lyapunov functionals. Moreover, stronger results for the case where the matrix multiplying the delayed state is itself subject to delay are also given in [18].

\subsection{Multiple delays}

Finally, for this section, consider the family of subsystems

$$
\begin{aligned}
x(k+1)= & A^{(s)} f(x(k))+B_{1}^{(s)} f(x(k-1))+\cdots \\
& +B_{l}^{(s)} f(x(k-l)),
\end{aligned}
$$

where $A^{(s)}, B_{1}^{(s)}, \ldots, B_{l}^{(s)}$ are nonnegative matrices for $1 \leq s \leq N$, and the nonlinearities $f(x)=\left(f_{1}\left(x_{1}\right), \ldots, f_{n}\left(x_{n}\right)\right)^{T}$ satisfy the conditions (8), (9).

It is relatively straightforward, although the notation becomes more opaque, to adapt the argument used in the proof of Theorem 5.1 to obtain the following result for systems with multiple delays.

Theorem 5.2. Assume there exist positive vectors $\theta \gg 0, d \gg 0$ satisfying the inequalities

$$
\begin{aligned}
& \left(A^{(s)}+B_{1}^{\left(\nu_{1}\right)}+\cdots+B_{l}^{\left(\nu_{l}\right)}-I\right)^{T} d \ll 0, \\
& \quad s, v_{1}, \ldots, v_{l}=1, \ldots, N, \\
& \left(A^{(s)}+B_{1}^{(s)}+\cdots+B_{l}^{(s)}-I\right) \theta \ll 0, \quad s=1, \ldots, N .
\end{aligned}
$$

Then there exist positive definite diagonal matrices $P, Q_{1}, \ldots, Q_{l}$ such that (11) defines a common $L-K$ functional for the family (18).

Example 5.2. Consider a linear example of the system (13) (setting $f_{i}$ to be the identity for all $i$ ), with $N=2$ and

$$
\begin{aligned}
A_{1}=\left(\begin{array}{ll}
0.5 & 0.2 \\
0.3 & 0.1
\end{array}\right), & B_{1}=\left(\begin{array}{ll}
0.1 & 0.1 \\
0.2 & 0.1
\end{array}\right) ; \\
A_{2}=\left(\begin{array}{ll}
0.3 & 0.3 \\
0.1 & 0.2
\end{array}\right), & B_{2}=\left(\begin{array}{cc}
0.15 & 0.1 \\
0.1 & 0.15
\end{array}\right) .
\end{aligned}
$$

It can be verified that the conditions (14), (15) are satisfied with $d=\theta=(3,2)^{T}$. It follows that there exists a diagonal L-K functional for the associated switched time-delay system. In general, verifying the existence of solutions to (14), (15) can be done using techniques from linear programming (essentially it is a feasibility problem) or applying results such as those described in [6].

\section{Conclusions}

Building on recent work on the existence of diagonal L-K functionals for delayed positive linear systems in continuous-time, we have derived corresponding results for discrete-time positive systems. Moreover, we have extended these to give conditions for the existence of diagonal-type Lyapunov functionals for a class of nonlinear positive systems, which are not necessarily orderpreserving. The problem of common $\mathrm{L}-\mathrm{K}$ functional existence for switched positive systems has also been addressed. We have provided a sufficient condition for such a functional to exist. An interesting question for the future is to investigate the relationship between the existence of common Lyapunov functions for switched systems with zero delay and the corresponding problem for L-K functionals for delayed systems. As indicated in Example 5.1, this relationship is in general not straightforward.

\section{Acknowledgments}

The authors would like to thank the anonymous reviewers and the handling editor for their helpful and constructive remarks, which have helped to improve the overall presentation.

The second author was supported by the Irish Higher Educational Authority (HEA) PRTLI 4 Network Mathematics Grant. The first author was supported by the St. Petersburg State University, project no. 9.38.674.2013, and by the Russian Foundation of Basic Researches (RFBR), grant nos. 13-01-00376-a and 13-08-00948-a.

\section{References}

[1] L. Farina, S. Rinaldi, Positive Linear Systems. Theory and Applications, in: Pure and Applied Mathematics, John Wiley \& Sons, Inc., 2000.

[2] T. Kaczorek, Positive 1D and 2D Systems, Springer-Verlag, 2002.

[3] E. Fornasini, M.E. Valcher, Stability and stabilizability criteria for discretetime positive switched systems, IEEE Trans. Automat. Control 57 (5) (2012) 1208-1221.

[4] F. Blanchini, P. Colaneri, M.E. Valcher, Co-positive Lyapunov functions for the stabilization of positive switched systems, IEEE Trans. Automat. Control 57 (12) (2012) 3038-3050.

[5] A.Yu. Aleksandrov, Y. Chen, A.V. Platonov, L. Zhang, Stability analysis for a class of switched nonlinear systems, Automatica 47 (10) (2011) 2286-2291.

[6] F. Knorn, O. Mason, R. Shorten, On copositive linear Lyapunov functions for sets of linear positive systems, Automatica 45 (8) (2009) 1943-1947.

[7] P.H.A. Ngoc, Stability of positive differential systems with delay, IEEE Trans. Automat. Control 58 (1) (2013) 203-208.

[8] V.S. Bokharaie, O. Mason, M. Verwoerd, D-stability and delay-independent stability of homogeneous cooperative systems, IEEE Trans. Automat. Control 55 (12) (2010) 1996-2001.

[9] O. Mason, Diagonal Riccati stability and positive time-delay systems, Systems Control Lett. 61 (1) (2012) 6-10.

[10] E. Kaszkurewicz, A. Bhaya, Matrix Diagonal Stability in Systems and Computation, Birkhäuser, 2000.

[11] W.M. Haddad, V. Chellaboina, Stability theory for nonnegative and compartmental dynamical systems with time-delay, Systems Control Lett. 51 (5) (2004) 355-361.

[12] J. Hofbauer, J.W. So, Diagonal dominance and harmless off-diagonal delays, Proc. Amer. Math. Soc. 128 (2000) 2675-2682.

[13] A.Yu. Aleksandrov, A.V. Platonov, Aggregation and stability analysis of nonlinear complex systems, J. Math. Anal. Appl. 342 (2) (2008) 989-1002.

[14] P.J. Psarrakos, M.J. Tsatsomeros, A primer of Perron-Frobenius theory for matrix polynomials, Linear Algebra Appl. 393 (2004) 333-351.

[15] L. Wu, J. Lam, Z. Shu, B. Du, On stability and stabilizability of positive delay systems, Asian J. Control 11 (2) (2009) 226-234.

[16] W. Lombardi, S. Olaru, G. Bitsoris, S.-I. Niculescu, Cyclic invariance for discrete time-delay systems, Automatica 48 (2012) 2730-2733.

[17] M. Buslowicz, Simple stability conditions for linear positive discrete-time systems with delays, Bull. Pol. Acad. Sci., Tech. Sci. 56 (4) (2008) 325-328.

[18] A. Zappavigna, P. Colaneri, J.C. Geromel, R. Shorten, Dwell time analysis for continuous-time switched linear positive systems, in: Proceedings of the American Control Conference, 2010, pp. 6256-6261. 